\title{
A hardware implementation of random demodulation analog-to-information converter
}

\author{
Wenbo Xu ${ }^{1 \text { a) }}$, Yupeng $\mathrm{Cui}^{1}$, Yue Wang ${ }^{2}$, \\ Siye Wang ${ }^{1}$, and Jiaru Lin ${ }^{1}$ \\ ${ }^{1}$ Key Laboratory of Universal Wireless Communication, Ministry of Education, \\ Beijing University of Posts and Telecommunications, Beijing 100876, China \\ ${ }^{2}$ Hisilicon Research Department, Huawei Technologies Co. Ltd, Beijing, China \\ a)xuwb@bupt.edu.cn
}

Abstract: The analog-to-information converter (AIC) based on compressed sensing samples the analog signals at sub-Nyquist rate. This paper presents a low-cost hardware implementation of AIC based on random demodulation. The realization consists of a sampling part and a transmission part, which are connected by using a FPGA chip. In the former, the input analog signal is modulated with a pseudo-random sequence, and sampled with sub-Nyquist rate to obtain low-dimensional measurements. In the latter, the measurements are transmitted with user datagram protocol to the computer. The experimental results demonstrate the effectiveness of the hardware implementation. Keywords: compressed sensing, analog-to-information converter, random demodulation, hardware platform

Classification: Electron devices, circuits and modules

\section{References}

[1] E. J. Candes, et al.: "Stable signal recovery from incomplete and inaccurate measurements," Commun. Pure Appl. Math. 59 (2006) 1207 (DOI: 10.1002/ cpa.20124).

[2] S. Kirolos, et al.: "Analog-to-information conversion via random demodulation," 2006 IEEE Dallas/CAS Workshop on Design, Applications, Integration and Software (2006) 71 (DOI: 10.1109/DCAS.2006.321036).

[3] M. Mishali and Y. C. Eldar: "From theory to practice: sub-Nyquist sampling of sparse wideband analog signals," IEEE J. Sel. Topics Signal Process. 4 (2010) 375 (DOI: 10.1109/JSTSP.2010.2042414).

[4] J. Yoo, et al.: "Design and implementation of a fully integrated compressedsensing signal acquisition system," ICASSP, IEEE Int. Conf. Acoust. Speech Signal Process (2012) 5325 (DOI: 10.1109/ICASSP.2012.6289123).

[5] W. Xu, et al.: "Performance analysis of partial segmented compressed sampling," Signal Process. 93 (2013) 2653 (DOI: 10.1016/j.sigpro.2013.02.001).

[6] R. T. Yazicigil, et al.: "19.4 A 2.7 -to-3.7 GHz rapid interferer detector exploiting compressed sampling with a quadrature analog-to-information converter," IEEE International Solid-State Circuits Conference - (ISSCC) Digest of Technical Papers (2015) 1 (DOI: 10.1109/ISSCC.2015.7063069). 
[7] J. Yoo, et al.: "A $100 \mathrm{MHz}-2 \mathrm{GHz} 12.5 \times$ sub-Nyquist rate receiver in $90 \mathrm{~nm}$ CMOS," IEEE Radio Frequency Integrated Circuits Symposium (2012) 31 (DOI: 10.1109/RFIC.2012.6242225).

[8] S. Liu, et al:: "Theory and hardware implementation of an analog-toinformation converter based on compressive sensing," IEEE 10th International Conference on ASIC (ASICON) (2013) 1 (DOI: 10.1109/ASICON.2013. 6812033).

[9] T. Murray, et al:: "Design of a CMOS A2I data converter: theory, architecture and implementation," 45th Annual Conference on Information Sciences and Systems (2011) 1 (DOI: 10.1109/CISS.2011.5766231).

[10] A. Dutta and R. K. Mangang: "Analog to information converter based on random demodulation," International Conference on Electronic Design, Computer Networks \& Automated Verification (EDCAV) (2015) 105 (DOI: 10.1109/EDCAV.2015.7060548).

[11] J. N. Laska, et al.: "Theory and implementation of analog-to-information converter using random demodulation," IEEE International Symposium on Circuits and Systems (2007) 1959 (DOI: 10.1109/ISCAS.2007.378360).

[12] J. Tropp and A. Gilbert: "Signal recovery from random measurements via orthogonal matching pursuit," IEEE Trans. Inf. Theory 53 (2007) 4655 (DOI: 10.1109/TIT.2007.909108).

\section{Introduction}

The theory of compressed sensing (CS) exploits the sparse prior to represent the signals with fewer measurements than the number stated in the Nyquist theorem [1]. According to CS theory, these few measurements are obtained by multiplying signals with a measurement matrix. To obtain compressed measurements directly from an analog signal at sub-Nyquist rate, analog-to-information converter (AIC) has been proposed as a practical technique.

Currently, many AIC architectures have been designed in the literature, such as random demodulation AIC (RD-AIC) [2], modulated wideband converters (MWC) [3], random-modulation pre-integrator (RMPI) [4], partial segmented AIC [5], and so on. Unfortunately, although many works discussed the structures of AIC, only few works discussed the practical hardware implementation, e.g., $[4,6,7,8,9$, 10, 11]. [6] proposed the hardware implementation of an AIC scheme based on MWC, and [4, 7] presented the hardware platforms of RMPI. For RD-AIC, [8, 9, $10,11]$ described the theory and hardware implementation; however, they only gave the simulated results.

In this paper, we realize a simple and low-cost hardware platform of RD-AIC structure. The platform, controlled by FPGA, consists of a sampling part and a transmission part. The sampling part is used to convert the input analog signal to low-dimensional digital measurements, and the transmission part sends the measurements through the Ethernet to a computer to reconstruct the original signal. We show the performance of our hardware platform for both the single-tone signal and the multi-tone signal. The reconstruction results demonstrate that the input analog sparse signals can be reconstructed reliably with a very low sampling rate, which is $1 / 10$ times of the traditional Nyquist rate. This proportion is superior to the $1 / 4$ in the recent work [10]. 


\section{Backgrounds}

Compressed sensing deals with the acquisition and recovery of sparse signals from a small number of random linear measurements. In the acquisition phase, a $K$ sparse signal $\mathbf{x} \in \mathcal{R}^{N}$ can be represented by its fewer measurements in the form of

$$
\mathbf{y}=\boldsymbol{\Phi} \mathbf{x}
$$

where $\mathbf{y} \in \mathbb{R}^{M}$ is the measurement vector, and $\mathbf{\Phi} \in \mathbb{R}^{M \times N}$ is the measurement matrix $(K<M<N)[1]$.

In the recovery phase, the original signal $\mathbf{x}$ is recovered from $\mathbf{y}$ based on the prior information of signal sparsity. [1] showed that the sparse signal can be reliably recovered under some conditions on the measurement matrix $\boldsymbol{\Phi}$ by solving an $l_{1}$ minimization problem. Many other recovery algorithms are also reported thereafter. Among them, orthogonal matching pursuit (OMP) [12] is a popular greedy algorithm, which has been used widely.

\section{Practical implementation}

\subsection{Hardware architecture}

Based on the theoretical structure in [2], we implement RD-AIC hardware architecture as shown in Fig. 1, which obtain digital measurements directly from analog signals. The proposed architecture consists of a sampling part, a transmission part, and a FGPA chip, where the FPGA connects the first two parts.

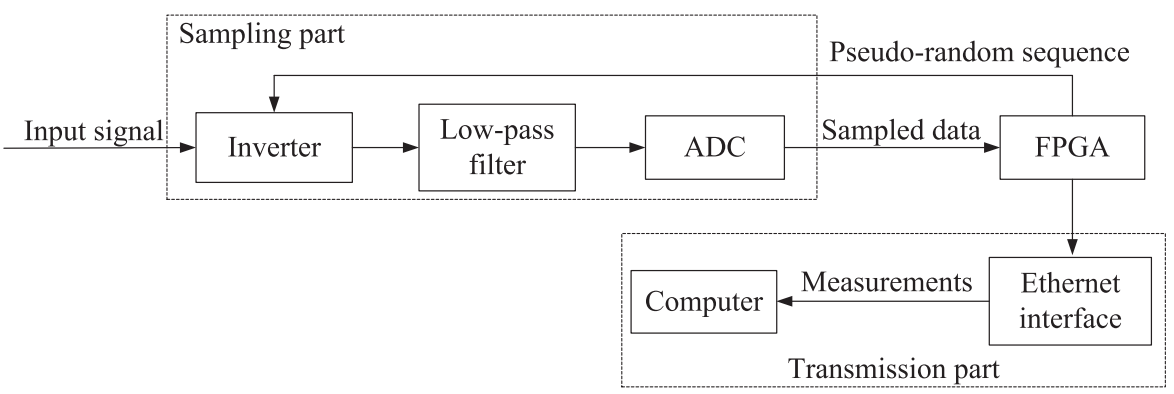

Fig. 1. RD-AIC architecture

The sampling part includes an inverter, a low-pass filter and an analog-to-digital converter (ADC), where the ADC works with a sub-Nyquist rate and outputs the low-dimensional measurements. The inverter is used to realize the demodulation with a pseudo-random sequence, which spreads the frequency content of the signal. By using an inverter as shown in Fig. 2 rather than a multiplier, the hardware cost is greatly reduced. The inverter is implemented by a pair of controlled switches, where the two switches are controlled synchronously by the pseudo-random sequence that is generated by FPGA, but their states are always opposite. Specifically, when the incoming bit of pseudo-random sequence is positive, the positive switch is on, and the negative switch is connected to the ground. Then signal is input through the upper branch in Fig. 2, and vice versa. The low-pass filter is used to avoid the frequency aliasing phenomenon, and the cut-off frequency is set to be $5 \mathrm{MHz}$. In this design, we used a 5 order $\pi$ type LC filter. The filtered results will be 
sampled with $10 \mathrm{MHz}$ sampling rate and quantized with 14 bits by ADC to obtain the measurements.

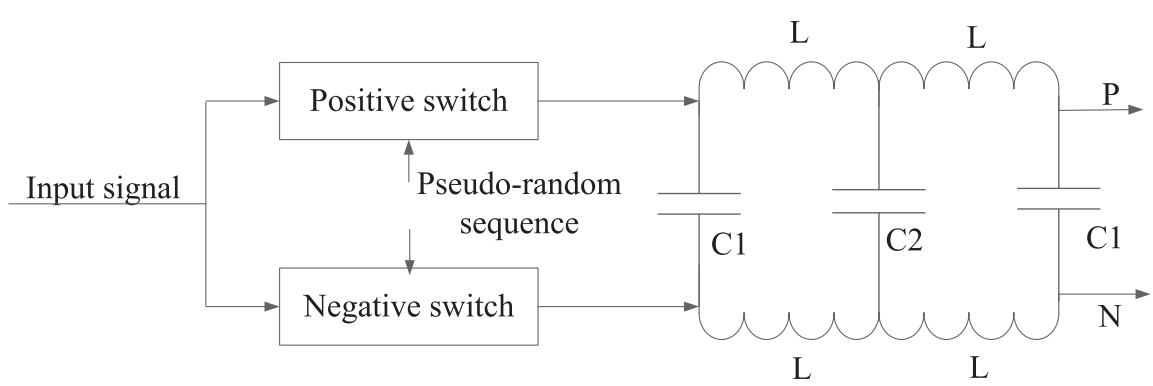

Fig. 2. The implementation of inverter and filter

The FPGA buffers the obtained measurements and divides them into frames. Then, it implements the communication protocol of ethernet and transmits the measurements with user datagram protocol (UDP) frames. The transmission part sents the buffered frames to the computer through the Ethernet, and finally the original signal is reconstructed at the computer with the received measurements.

The parameters of the system are shown in Table I. Note that the sampling rate is only $1 / 10$ times of the Nyquist sampling rate. In addition, Fig. 3 gives the photo of our realized hardware platform.

Table I. Parameters of the system

\begin{tabular}{lc}
\hline system clock & $125 \mathrm{MHz}$ \\
\hline bandwidth of input signal & $50 \mathrm{MHz}$ \\
\hline rate of pseudo-random sequence & $100 \mathrm{MHz}$ \\
\hline sub-Nyquist sampling rate & $10 \mathrm{MHz}$ \\
\hline number of quantization bits & 14 \\
\hline length of measurements in one frame & $M=100$ \\
\hline length of reconstructed signal in one frame & $N=1000$ \\
\hline
\end{tabular}

\subsection{System model}

Suppose that the input signal $s(t)$ is sparse in continuous fourier basis, which can be expressed as

$$
s(t)=\sum_{n=1}^{N} \alpha_{n} \Psi_{n}(t)
$$

where $\Psi_{n}(t)$ represents the continuous fourier basis function and $\alpha_{n}$ is the sparse coefficient. Let $p_{c}(t)$ stand for the pseudo-random sequence and $h(t)$ the impulse response of the low-pass filter. Then, for any frame, we can write the $m$ th measurement $(m=1, \cdots, M)$ as

$$
y_{m}=\sum_{n=1}^{N} \alpha_{n} \int_{-\infty}^{+\infty} \Psi_{n}(\tau) p_{c}(\tau) h(T m-\tau) \mathrm{d} \tau
$$




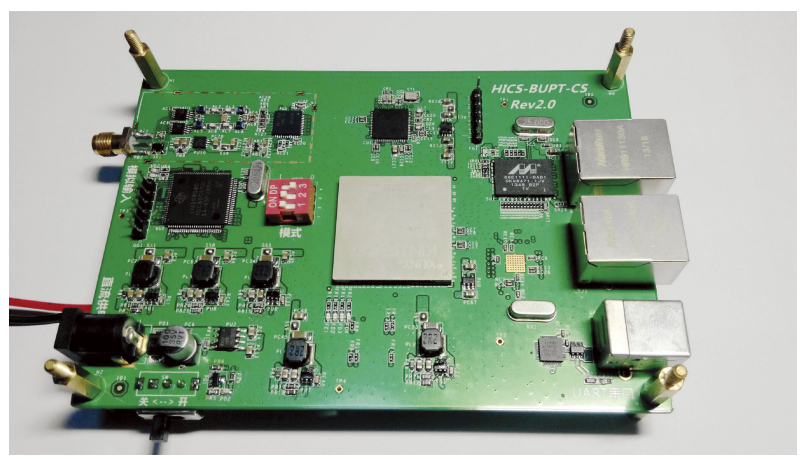

Fig. 3. Hardware platform

where $T$ stands for the sampling period in ADC. According to [2], by defining the measurement matrix $\mathbf{V}$ with the entries as $v_{m, n}=\int_{-\infty}^{+\infty} \Psi_{n}(\tau) p_{c}(\tau) h(t-\tau) \mathrm{d} \tau$, we obtain

$$
\mathbf{y}=\mathbf{V} \alpha
$$

where $\mathbf{y}=\left[y_{1}, y_{2}, \cdots, y_{M}\right]^{T}$ is the measurement vector, and $\alpha=\left[\alpha_{1}, \alpha_{2} \ldots \alpha_{n}\right]^{T}$ is the coefficient vector. Thus, the reconstruction of $\alpha$, and thus the input signal $s(t)$, from the measurements $\mathbf{y}$ can be achieved by using any CS reconstruction algorithms. In this paper, we use OMP as the reconstruction algorithm.

\section{Experimental results}

To verify the performance of the implemented hardware platform, we first input a single-tone signal with frequency $42.1 \mathrm{MHz}$. Fig. 4 shows the reconstructed signal in frequency domain, where the nonzero position is reconstructed successfully, and the spurious free dynamic range is obtained as $10.18 \mathrm{~dB}$. Note that by using other recovery algorithms with higher precision, the reconstruction performance can be further improved.

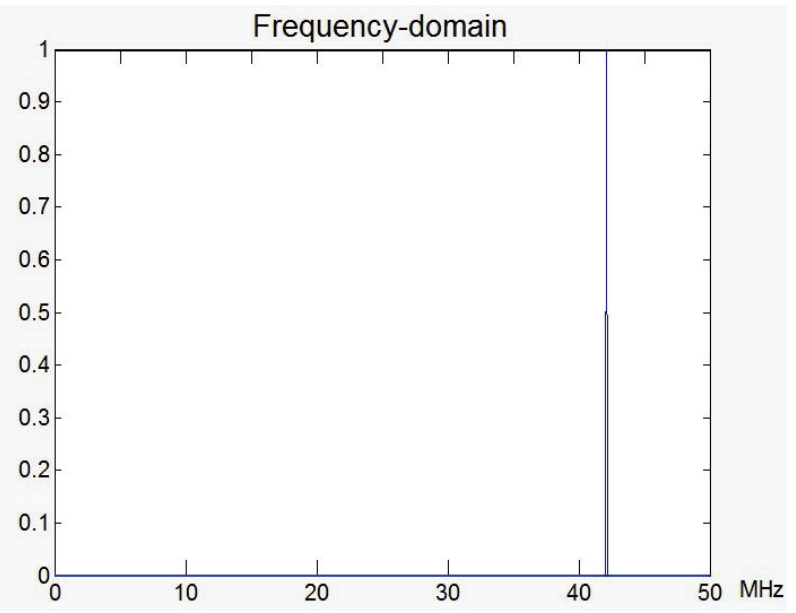

Fig. 4. Reconstruction result of single-tone signal 


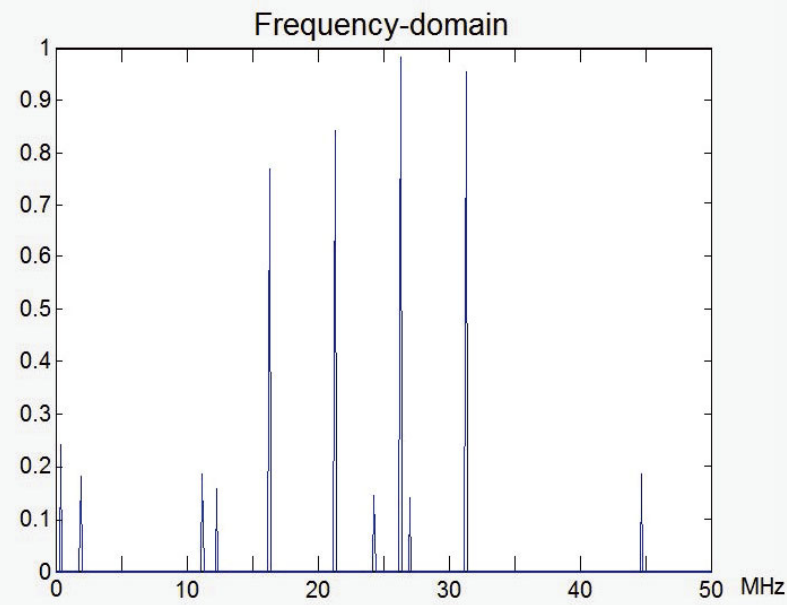

Fig. 5. Reconstruction result of multi-tone signal

platform. The nonzero positions are reconstructed successfully, however, the corresponding amplitudes are not equal. We note that such error can also be reduced by using reconstruction algorithms with higher quality.

\section{Conclusion}

In this paper, we describe a hardware implementation of the RD-AIC scheme, which is simple and low-cost. Using inverter, filters, ADC and FPGA, the implemented RD-AIC hardware architecture enables the sparse analog signal to be sampled at a sub-Nyquist rate directly. Experimental results show that the hardware platform reconstructs the nonzero positions correctly for both the single-tone and multi-tone signals. However, further efforts are needed to perfectly recover the amplitudes. In addition, currently we reconstruct the signals at computer, one future work is to realize the practical hardware platform for reconstruction.

\section{Acknowledgments}

This work was supported by the National Natural Science Foundation of China (61302084), Huawei Innovation Research Program under Grant YB2014010044, and the Fundamental Research Funds for the Central Universities (2015RC02). 